\title{
Influence of the Voltage on Pore Diameter and Growth Rate of Thin Anodic Aluminium Oxide (AAO) Pattern on Silicon Substrate
}

\author{
Khaled M. Chahrour*, Poh Choon Ooi, Azrul Azlan Hamzah \\ Institute of Microengineering and Nanoelectronics, Universiti Kebangasaan Malaysia, 43600, Selangor-Malaysia
}

\section{Article information}

\section{Article history:}

Received: June, 09, 2021

Accepted: June, 24, 2021

Available online: June, 25, 2021

Keywords:

AAO pattern,

Anodization process,

Pore nanostructure,

Growth rate of pores

*Corresponding Author:

Khaled M. Chahrour

khaledmnchahrour@ukm.edu.my

skhaled_66@yahoo.com

\begin{abstract}
By applying dual anodization procedures of aluminum (Al) thin film over silicon substrate under controlled anodizing process conditions, a thin anodic alumina (AAO) pattern with hexagonal honeycomb-like arrangement nanopores and highly-regular aligned arrays was created. Anodizing DC voltage was thought to have an effect on pore size dimensions. With anodizing DC voltage, the pore diameter $(30-110 \mathrm{~nm})$ showed a linear change. The rate of vertical growth of the nanopores was found to be proportional to the anodizing voltage. To achieve open-through pore nanostructure and ensure homogeneous electrochemical deposition of various nanostructures onto AAO pattern, a bottommost barrier layer of the AAO pattern was detached during dropping the DC voltage in the final period of the anodization process, followed by a pore widening wet treatment for numerous minutes at ambient temperature atmosphere. The features of the AAO pattern were inspected by using the field emission scanning microscope (FESEM) linked with an electron dispersive $\mathrm{x}$ ray spectrometer (EDX) for chemical elements investigation.
\end{abstract}

DOI: 10.53293/jasn.2021.3801.1044, Department of Applied Science, University of Technology

This is an open access article under the CC BY 4.0 License

\section{Introduction}

Porous anodic alumina films (AAO) are self-ordered nanopore arrays created by the anodization of aluminium in a moderate acidic electrolyte using a DC power source; it's a favourable template for preparation of highly-regular nanostructure arrays. Due to its special qualities such as controlled pore diameter and periodicity, self-ordered nanopore arrays were used as an economical and effective approach in manufacturing nanostructure material, similar to lithography [1]. During the anodizing process, the nano-pore structures sprout perpendicular to the surface of the aluminium, with equilibrium between alumina layer creation at the metal/oxide boundary and alumina layer dissolution at the oxide/electrolyte boundary [2]. Controllable variations in anodizing conditions, such as the anodizing DC voltage, the interval of anodization time, the concentration, and the temperature of the electrolyte, can be used to change the size, density, and thickness of the pores $[3,4]$. Ono S. et al establish that self-ordered pore arrays could take place at an anodizing DC voltage corresponding to a high electric field [5]. Based on their consequences, they recommended that a high electric field condition is the most important controlling factor governing the self-ordering process. However, the formation mechanism of the ordered pore arrays, especially the relation between well-ordered pore formation and anodizing process conditions, has not been fully confirmed. The goal of this research is to investigate the effect of anodic voltage on the formation of highly ordered pore arrays on aluminium thin films with a thickness of just $(1 \mu \mathrm{m})$, as well as to establish a relationship between the vertical development rates of the pores and anodic voltage. 


\section{Experimental details}

Thin and high-quality aluminium (Al) film on silicon substrate was completed by applying an evaporator with a high-vacuum e-beam. The materials that were used here is Si wafer oriented in the p-type (100) direction. Highpurity $\mathrm{Al}$ pellets are used as the $\mathrm{Al}$ source $(99.98 \%)$. A homogeneous deposited Al metal layer with a thickness of up to $1.0 \mu \mathrm{m}$ can be achieved in one continuous run. In the instance of the $1.0 \mu \mathrm{m} \mathrm{Al}$ metal film, a thin layer $(30 \mathrm{~nm})$ of Ti metal was pre-deposited between the Al metal film and the Si substrate to provide strong adhesion properties during the anodization process. A dual step approach was used to anodize the evaporated $\mathrm{Al}$ film in a $0.3 \mathrm{M}$ oxalic acid aqueous solution $[6,7]$. The anodizing process was performed under the time of the first-step of anodization is $10 \mathrm{~min}$ and the temperature at $19{ }^{\circ} \mathrm{C}$ in a unique electrochemical Teflon cell design utilizing a platinum rod as the cathode and the Al/Si substratum as the anode as shown in the schematic diagram Fig. 1. The anodic voltage was controlled using a Keithley source metre model 2400 to provide well-ordered arrays of nanopores with consistent pore diameter. Applied DC voltages were 35, 45, and 55V, respectively. After the firststep of anodizing process, AAO layer was etched out in $6 \mathrm{wt} \%$ of phosphoric acid and $1.8 \mathrm{wt} \%$ of chromic acid at $70{ }^{\circ} \mathrm{C}$ for $30 \mathrm{~min}$. The second step of the anodizing process was carried out until all of the aluminium film had been thoroughly anodised under the same conditions as the first. The anodizing voltage was ramped at a rate of 1 $\mathrm{V} / \mathrm{s}$ throughout the final period of the second-step anodization, followed by 30 minutes of immersion in a $4 \mathrm{wt} \%$ phosphoric acid solution to remove the almost bottommost barrier layer. Field emission scanning electron microscopy (FESEM) and EDX analyses were used to analyse the structure and chemical composition of the AAO pattern.

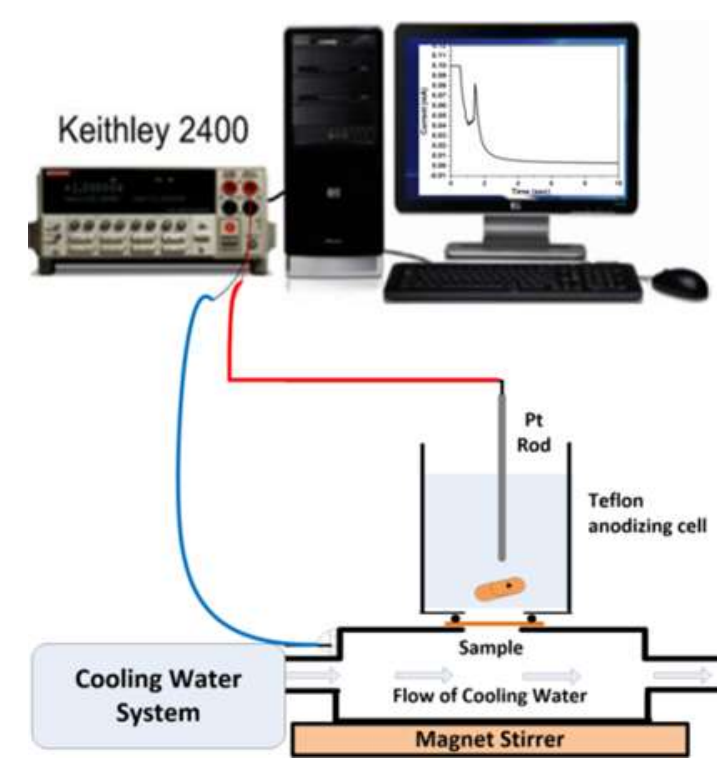

Figure: 1 Schematic diagram of experimental setup of anodization.

\section{Results and Discussion}

Dual step anodization in oxalic acid solution $(0.3 \mathrm{M})$ at a certain voltage was used to fully develop an AAO pattern. At the electrochemical cell electrodes, anodization occurs in two primary chemical processes [8]:

At the anode terminal: $2 \mathrm{Al}+\mathbf{3} \mathrm{H}_{2} \mathbf{O} \rightarrow \mathbf{A l}_{2} \mathbf{O}_{3}+\mathbf{6} \mathbf{H}^{+}+\mathbf{6 e}$

$$
\mathbf{A l}_{2} \mathbf{O}_{3}+6 \mathbf{H}^{+} \rightarrow \mathbf{2} \mathbf{A l}^{+3}+3 \mathbf{H}_{2} \mathbf{O} \ldots \ldots \ldots \ldots \text { (2) (Dissolution of Alumina) }
$$

At the Cathode terminal: $\quad \mathbf{6} \mathbf{H}^{+}+\mathbf{6 e} \rightarrow \mathbf{3} \mathbf{H}_{2}$

Figure 2 a, $\mathbf{b}$ and $\mathbf{c}$ shows a typical top view of the AAO patterns at 35, 45, and 55 volts respectively.

The results show that a two-step anodization technique can produce an ordered honeycomb-like nanostructure with uniform pore diameter and spacing under adjustable parameter circumstances. Figure $\mathbf{2}$ a, $\mathbf{b}$ and $\mathbf{c}$ illustrate that the pore diameter enlarge with rising DC voltage. The average of pore width is $32 \mathrm{~nm}, 76 \mathrm{~nm}$ and $105 \mathrm{~nm}$ for 
AAO pattern fabricated under DC voltage of $35 \mathrm{~V}, 45 \mathrm{~V}$ and $55 \mathrm{~V}$, respectively. A curve relating average pore diameter with anodizing DC voltage described in Figure $2 \mathbf{d}$ demonstrates that the average pore diameter is enlarged with increasing the DC voltage. Although, the mechanism of vertically nanopore creation is quiet not fully determined, it is usually established that the driving force create as of the DC voltage applying for the selfordering procedure can be ascribed to a stress-driven interface produced by the repulsive forces between adjacent nanopores which lead to perfect self-ordered. The changing stress created by volume expansion modifies the value of regularity and drives the perfect cylindrical and hexagonal honeycomb-like arrangement of nanopores in the self-assembly production of the AAO pattern [9].
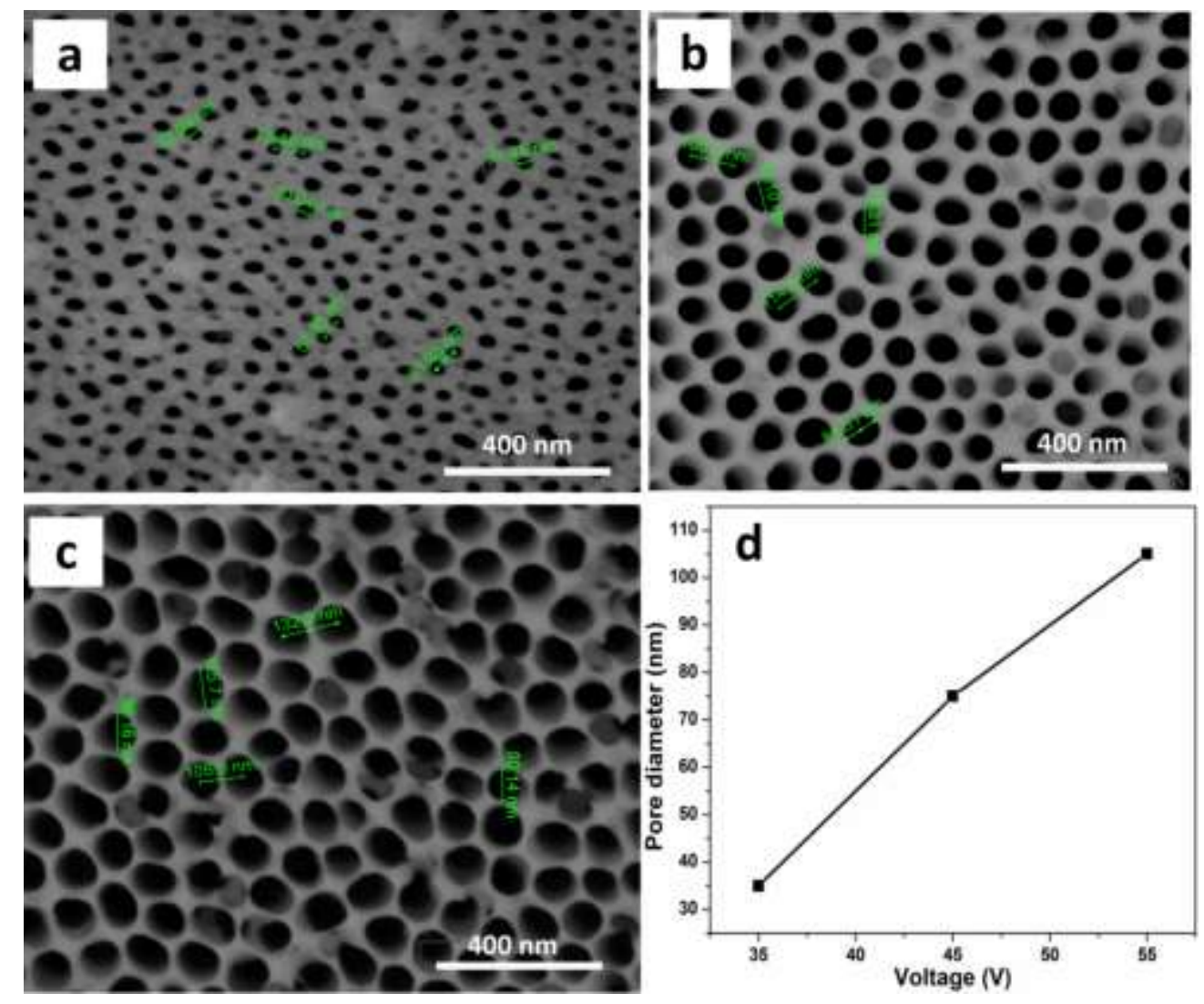

Figure 2: (a), (b) and (c), The AAO patterns are seen in top view in FESEM images. AAO patterns were made under the following conditions: first-step anodization acquired 10 minutes, pore widening acquired 30 minutes, and anodizing voltages were 35,45 , and $55 \mathrm{~V}$, respectively. Figure (d) illustrates the pore diameter as a function of voltage.

Figure 3 a, b and $\mathbf{c}$ show cross-sectioned view of the AAO patterns for different DC voltages of anodization under 35,45 , and $55 \mathrm{~V}$, respectively. The results display clearly that the thickness of the pattern can be adjusted by changing the anodizing voltage. Figure $3 \mathrm{~d}$ depicts the curve of the AAO pattern's growth rate $\left(\mathrm{R}_{\mathrm{p}}\right)$ as a function of voltage. The semi-logarithmic plots of $R_{p}$ as a linear variation with DC voltage is shown in the upper inset of figure $3 \mathrm{~d}$, indicating that $\mathrm{R}_{\mathrm{p}}$ changes exponentially with anodizing voltage. These results corresponded with results of Hwang, S.K. [10]. 

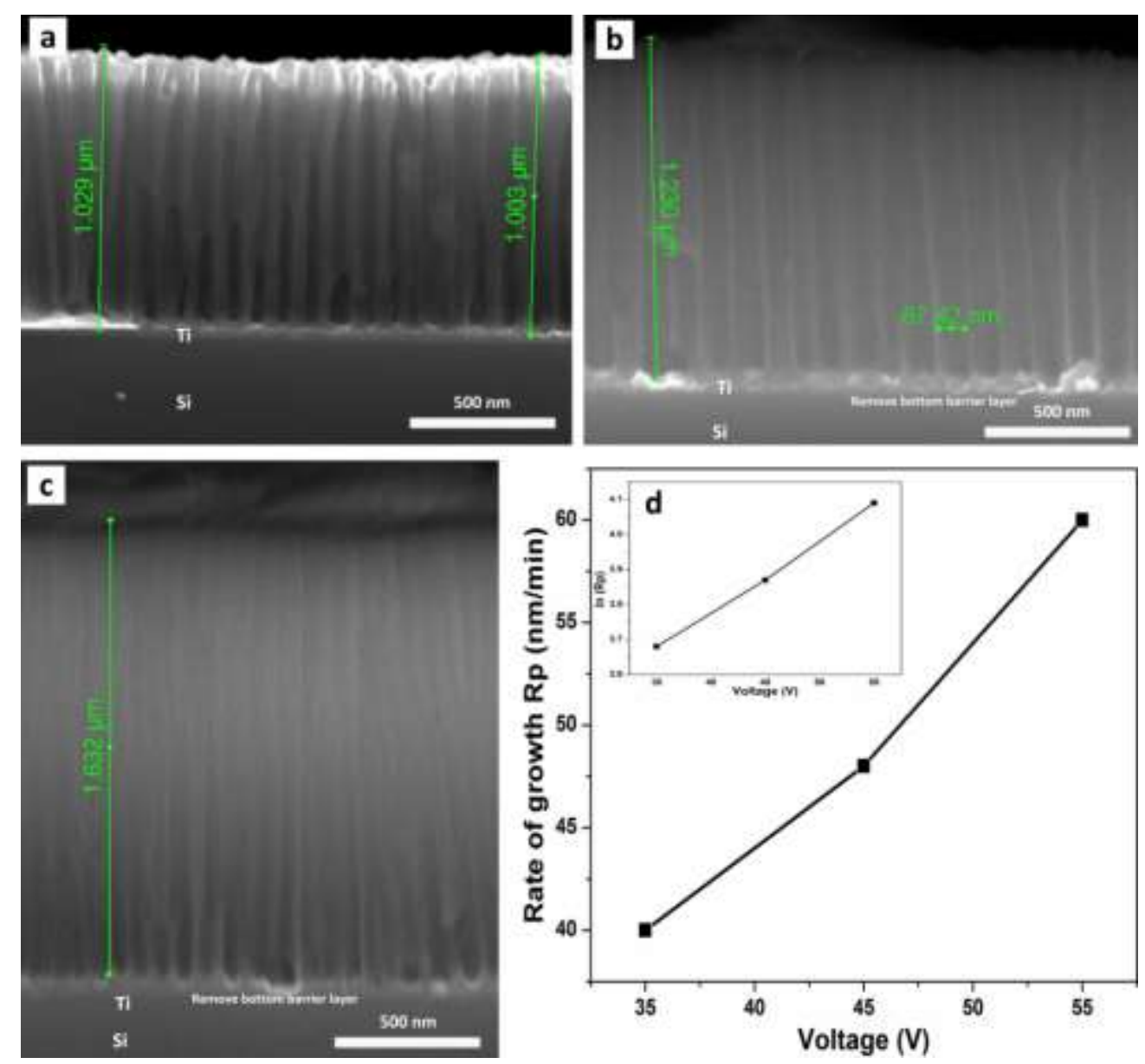

Figure 3: (a), (b) and (c) show FESEM images cross-section of the AAO patterns were prepared under anodizing voltage 35, 45, $55 \mathrm{~V}$, respectively. (d) Depicts the growth rate curve as a function of anodizing. The semilogarithmic plot of growth rate as a function of anodizing voltage is shown in the upper inset.

Figure 4 demonstrates the documented EDX spectrum validating the chemical compositions of the cross-segment view of AAO pattern onto Ti/Si substratum were analogous for various anodizing voltages.

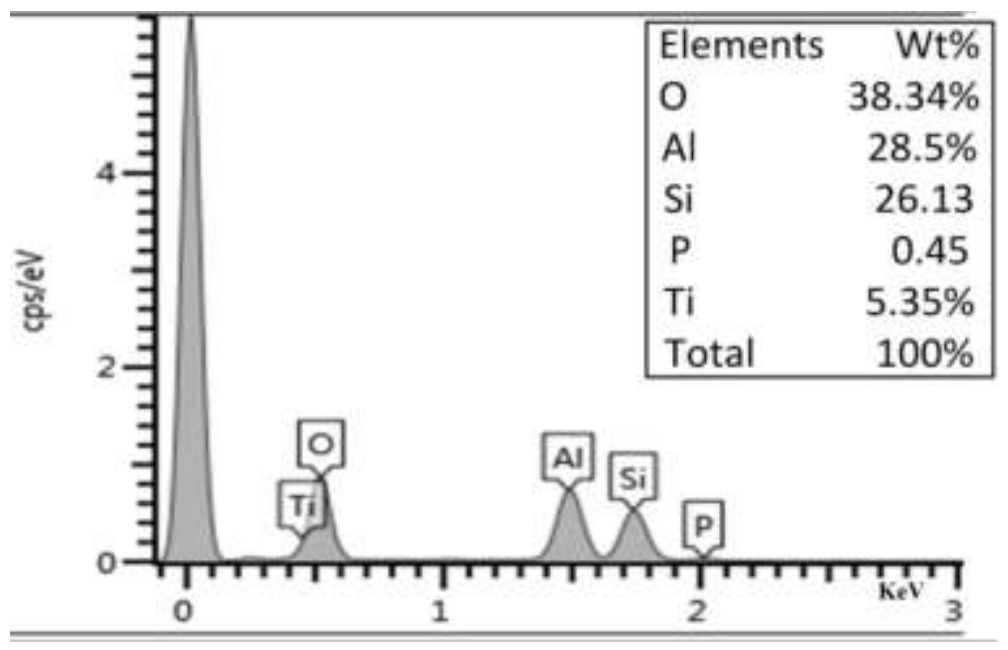

Figure 4: EDX spectrum of AAO template on Ti/Si substrate. 
To achieve open-through pore nanostructure, the alumina barrier layer, which is the bottommost of the Ti/Si based AAO nanotubes, was eliminated by reducing the voltage in the final period of the anodization process, followed by a pore widening treatment for several minutes at ambient atmosphere. This process performed at room temperature. In literature, previous literatures used chemical etching process for removing alumina barrier layer with simple acidic etching at a particular temperature [11]. Furthermore, the removal of the aluminium oxide barrier layer on the bottom of the AAO pore arrays, which increases the electric contact between the substrate and the AAO, is enabling uniform electrochemical deposition of diverse nanostructures onto the AAO pattern. [12].

\section{Conclusions}

Dual stage anodization of thin Al film $(1 \mu \mathrm{m})$ over Si substrate under manageable anodizing parameters resulted in highly regular hexagonal honeycomb-like nanopore arrays in AAO patterns with same pore size and vertically aligned nanotubes. Anodizing voltage contributed considerable positive effect on pore diameters, according to FESEM investigations. To generate an open-through pore nanostructure, the voltage was ramped and a straightforward wet etching technique for eliminating the bottom barrier layer of the AAO pattern was undertaken over several minutes.

\section{Acknowledgement}

The first author expresses gratitude to Institute of Microengineering and Nanoelectronics (IMEN), Universiti Kebangasaan Malaysia for awarding the Post-Doctoral Fellowship.

\section{Conflict of Interest}

The authors declare that they have no known competing financial interests or personal relationships that could have appeared to influence the work reported in this paper.

\section{References}

[1] J. Zhang, J. E. Kielbasa, and D. L. Carroll, "Controllable fabrication of porous alumina templates for nanostructures synthesis," Materials Chemistry and Physics, vol. 122, p. 295-300, 2010.

[2] D. Ma, S. Li, and C. Liang, "Electropolishing of high-purity aluminium in perchloric acid and ethanol solutions," Corrosion Science, vol. 51, p. 713-718, 2009.

[3] A. Belwalkar, E. Grasing, W. Van Geertruyden, Z. Huang, and W. Misiolek, "Effect of processing parameters on pore structure and thickness of anodic aluminum oxide (AAO) tubular membranes," Journal of membrane science, vol. 319, p. 192-198, 2008.

[4] K. M. Chahrour, N. M. Ahmed, M. Hashim, N. G. Elfadill, W. Maryam, M. Ahmad, et al., "Effects of the voltage and time of anodization on modulation of the pore dimensions of AAO films for nanomaterials synthesis," Superlattices and Microstructures, vol. 88, p. 489-500, 2015.

[5] S. Ono, M. Saito, M. Ishiguro, and H. Asoh, "Controlling factor of self-ordering of anodic porous alumina," Journal of the Electrochemical Society, vol. 151, p. B473, 2004.

[6] H. Masuda and K. Fukuda, "Ordered metal nanohole arrays made by a two-step," Science, vol. 268, p. 1466-1468, 1995.

[7] H. Masuda, H. Yamada, M. Satoh, H. Asoh, M. Nakao, and T. Tamamura, "Highly ordered nanochannelarray architecture in anodic alumina," Applied Physics Letters, vol. 71, p. 2770-2772, 1997.

[8] K. Chahrour, N. Ahmed, M. R. Hashim, N. Elfadill, and M. A. Qaeed, "Controllable fabrication of highly ordered thin AAO template on Si substrate for electrodeposition of nanostructures," Applied Physics A, vol. 116, p. 1389-1393, 2014.

[9] A. M. Md Jani, D. Losic, and N. H. Voelcker, "Nanoporous anodic aluminium oxide: Advances in surface engineering and emerging applications," Progress in Materials Science, vol. 58, p. 636-704, 2013.

[10] S.-K. Hwang, S.-H. Jeong, H.-Y. Hwang, O.-J. Lee, and K.-H. Lee, "Fabrication of highly ordered pore array in anodic aluminum oxide," Korean Journal of Chemical Engineering, vol. 19, p. 467-473, 2002. 
[11] N. Holubowitch, L. C. Nagle, and J. F. Rohan, "Porous alumina thin films on conductive substrates for templated 1-dimensional nanostructuring," Solid State Ionics, vol. 216, p. 110-113, 2012.

[12] K. M. Chahrour, F. Yam, N. M. Ahmed, M. Hashim, N. G. Elfadill, A. M. Al-Diabat, et al., "AAOAssisted Synthesis of Aligned CuO Nanorod Arrays by Electrochemical Deposition for Self-powered NIR Photodetection," Journal of Electronic Materials, vol. 48, p. 7465-7473, 2019. 